\title{
More TORC for the gluconeogenic engine
}

\author{
Alan Cheng and Alan R. Saltiel*
}

\begin{abstract}
Summary
Hepatic gluconeogenesis plays a key role in the maintenance of glucose homeostasis. The hormone glucagon stimulates this process, whereas insulin and adiponectin are inhibitory. In a recent report, Koo et al identify the transcriptional regulator TORC2 (Transducer of Regulated CREB activity 2) as a pivotal component of the gluconeogenic program. ${ }^{(1)}$ Both insulin and AMPK increase the phosphorylation of TORC2, while glucagon suppresses it. This in turn regulates the nuclear/cytoplasmic shuttling of TORC2 and its ability to transactivate gluconeogenic genes. Thus, TORC2 might serve as a gluconeogenic "molecular switch" that senses hormones and cellular energy status. BioEssays 28:231-234, 2006. ๑) 2006 Wiley Periodicals, Inc.
\end{abstract}

\section{Introduction}

The incidence of type 2 diabetes mellitus is reaching epidemic proportions, becoming one of the major causes of morbidity and mortality across the globe. Despite periods of feeding and fasting, in normal individuals plasma glucose remains in a narrow range. This tight control of glucose concentration is determined by a balance between glucose absorption from the intestine, production by the liver, and uptake in muscle and fat. ${ }^{(2)}$ Glucose production in the liver is controlled primarily by gluconeogenesis. ${ }^{(3)}$ The hormonal regulation of gluconeogenesis involves the transcriptional control of several key meta-

Departments of Internal Medicine and Physiology, Life Sciences Institute, University of Michigan Medical Center, Ann Arbor, Michigan ${ }^{*}$ Correspondence to: Alan R. Saltiel, Departments of Internal Medicine and Physiology, Life Sciences Institute, University of Michigan Medical Center, Ann Arbor, Michigan 48109, USA.

E-mail: saltiel@Isi.umich.edu

DOI 10.1002/bies.20375

Published online in Wiley InterScience (www.interscience.wiley.com).

Abbreviations: AMPK, Adenosine monophosphate activated protein kinase; cAMP, cyclic adenosine monophosphate; CBP, CREB binding protein; CREB, cAMP responsive element binding protein; G6Pase, Glucose-6-phosphatase; FOXA, Forkhead Box A; FOXO, Forkhead Box O; PEPCK, Phosphoenolpyruvate carboxykinase; PGC-1 $\alpha$, PPAR gamma coactivator-1 $\alpha$; PKA, Protein kinase A; SIK, Salt inducible kinase; SNF1, Sucrose nonfermenting 1; TORC, Transducer of regulated CREB activity. bolic enzymes, including phosphoenolpyruvate carboxykinase (PEPCK) and glucose-6-phosphatase (G6Pase). In the fasted state, glucagon is secreted, in turn increasing PEPCK and G6Pase levels, and elevating hepatic glucose output. In contrast, in the fed state, insulin inhibits the transcription of these genes, thereby shutting down gluconeogenesis.

\section{Gluconeogenesis and CREB}

The transcription factor CREB (CAMP Responsive Element Binding Protein) plays a critical role in the induction of gluconeogenic genes. ${ }^{(4)}$ Glucagon increases cAMP production, stimulating the phosphorylation of CREB at Ser133 by protein kinase $A(P K A)$. This subsequently induces the recruitment of transcriptional coactivators such as CREBbinding protein (CBP). Insulin opposes these effects, in part by increasing the phosphorylation of CBP at Ser436, thereby disrupting the CREB-CBP complex. ${ }^{(5)}$ The ability of CREB to regulate gluconeogenesis is largely dependent on the transcription factor PGC- $1 \alpha^{(6,7)}$ PGC- $1 \alpha$ is a transcriptional target of CREB, and its expression is triggered by elevated cAMP levels. PGC- $1 \alpha$ can also transactivate the PEPCK gene in response to glucocorticoids.

A recent search for additional coactivators of $C R E B$ yielded the family of TORC (transducer of regulated CREB activity) proteins. ${ }^{(8,9)}$ TORCs can potentiate the activity of both wildtype and phosphorylation-defective mutants of CREB. Although they interact with the DNA-binding/dimerization domain of CREB, they do not affect the ability of CREB to bind DNA. Instead, TORCs facilitate the ability of CREB to recruit the basal transcription machinery.

\section{TORCs: regulatory partners of CREB in gluconeogenesis}

Koo et $\mathrm{al}^{(1)}$ have elucidated the critical role TORC2 plays in the regulation of gluconeogenesis. Early studies paradoxically showed that both insulin and glucagon increased the phosphorylation of CREB at Ser133, suggesting that CREB phosphorylation alone is unable to distinguish between fasting and feeding signals. Nevertheless, insulin can also inhibit CREB-mediated gluconeogenesis through the phosphorylation of CBP, resulting in the disruption of the CREB-CBP complex. Koo et al examined mice with a knock-in mutation for CBP (CBP $\left.{ }^{k i x}\right)$ deficient for CREB binding. Despite the absence of CREB-CBP complexes, CBP ${ }^{\mathrm{kix}}$ mice displayed normal 
blood glucose levels, and responded to fasting with normal induction of gluconeogenic genes. Thus, the CREB-CBP complex alone is insufficient for the activation of the gluconeogenic program.

They then turned to the TORC proteins as a possible mechanism by which CREB mediates gluconeogenesis. Expression of TORC2 in primary hepatocytes dramatically increased the levels of PEPCK, G6Pase and PGC- $1 \alpha$ in response to elevated cAMP levels induced by forskolin. These effects were abrogated by co-expression of a dominant negative mutant of CREB, suggesting that the actions of TORC2 critically depend on CREB. Infection of mice with adenoviruses expressing TORC2 promoted fasting hyperglycemia. Conversely, infection with adenoviruses expressing TORC2 RNAi produced hypoglycemic mice with reduced expression of gluconeogenic genes during fasting. Thus TORC2 likely plays an important role in maintaining glucose metabolism through the control of hepatic gluconeogenesis.

\section{Phosphorylation of TORC: a molecular gluconeogenic switch}

The identification of TORC2 as an important player in the regulation of gluconeogenesis thus begs a key question, how is this protein itself regulated? TORC2 can be phosphorylated by the salt-inducible kinase 2 (SIK2) on Ser171, leading to its association with 14-3-3 proteins and sequestration to the cytoplasm from the nucleus. ${ }^{(10,11)}$ Moreover, cAMP inhibits SIK2 kinase activity through the PKA-dependent phosphorylation on Ser587 of SIK2. Consequently, dephosphorylation of TORC2 in response to CAMP results in nuclear translocation.

Koo et al explored in more detail how hormones might impact the subcellular localization of TORC2 through phosphorylation. In mice treated with insulin, TORC2 undergoes phosphorylation and is sequestered in the cytoplasm. Conversely, glucagon administration caused a dephosphorylation of TORC2 and relocalization to the nucleus, permitting the protein to now participate in transactivation of gluconeogenic genes. Thus TORC2 is able to distinguish between fasting and fed signals, and its phosphorylation may act as a molecular switch to regulate gluconeogenesis.

\section{Completing the regulatory circuit with SIK1}

It has long been known that the stimulatory effect of cAMP on PEPCK transcription is transient, ${ }^{(12)}$ leading Koo et al. to explore the temporal relationship of transcription to the phosphorylation state of TORC2. Treatment with the protein synthesis inhibitor cycloheximide abrogated Ser171 phosphorylation and increased the mRNA levels of gluconeogeneic genes and PGC-1 $\alpha$ suggesting a negative regulatory feedback loop that is dependent on de novo protein synthesis.

Unsurprisingly, SIK1 appears to be a key component of this negative feedback loop. The levels of SIK1, but not SIK2 or SIK3, were upregulated in liver upon fasting. Primary hepatocytes treated with forskolin responded similarly, and expression of TORC2 further potentiated SIK1 induction. Importantly, pretreatment with cycloheximide blocked the increase in SIK1 levels, demonstrating that SIK1 is indeed part of the inducible negative regulatory feedback loop on gluconeogenesis. Analysis of the SIK1 promoter region identified two cAMP response elements, and CREB constitutively occupied the promoter region by chromatin immunoprecipitation assays. Expression of PKA stimulated SIK1 promoter activity, whereas a dominant negative mutant of CREB blocked it. Treatment of cells with forskolin also increased promoter occupancy by TORC2 and phosphoCREB, further demonstrating the role of the TORC2-CREB pathway in the SIK1-mediated negative feedback loop.

The essential role of SIK was demonstrated in primary hepatocytes. SiRNA knockdown of SIK1 blocked glucagonstimulated TORC2 phosphorylation and further activated the gluconeogenic program. Similarly, depletion of SIK1 in fasted mice increased glucose levels and expression of gluconeogenic genes compared to controls, whereas infection of fasted mice with adenoviruses expressing SIK1 lowered fasting glucose levels and suppressed gluconeogenic gene expression. In primary hepatocytes, expression of SIK1 was able to block wild-type TORC2 but not its S171A mutant in stimulating PEPCK transcription. Taken together, these results demonstrate that SIK1 inhibits gluconeogenesis through the phosphorylation of TORC2.

\section{AMPK and TORC2}

SIK1 belongs to the SNF1/AMPK family of protein kinases. ${ }^{(13)}$ After noticing that Ser171 of TORC2 falls within an AMPK consensus substrate site, Koo et al demonstrated that it could also be phosphorylated by AMPK, resulting in cytoplasmic retention, while the localization of the S171A mutant of TORC2 was unaffected. Moreover, the S171A mutant partially rescued the AMPK-mediated suppression of PEPCK levels, suggesting that TORC2 is a critical downstream target of AMPK in the regulation of gluconeogenesis.

The hormones adiponectin and resistin reciprocally regulate gluconeogenesis by modulating the activation state of AMPK. ${ }^{(14,15)}$ It is likely that TORC2 is required for this effect. Furthermore, the widely prescribed diabetes drug Metformin has recently been shown to act through the LKB1-AMPKTORC2 pathway. ${ }^{(16)}$ Thus, TORC2 may represent a central switch to integrate signals from various hormones to regulate gluconeogenesis.

\section{Summary and future perspectives}

The studies by Koo et al further clarify our understanding as to how CREB and TORC2 operate to crank up the "gluconeogenic engine" (Fig. 1). During fasting, glucagon causes the nuclear shuttling of TORC2 to allow association with and coactivation of CREB on gluconeogenic genes. This involves

\section{BioEssays 28.3}




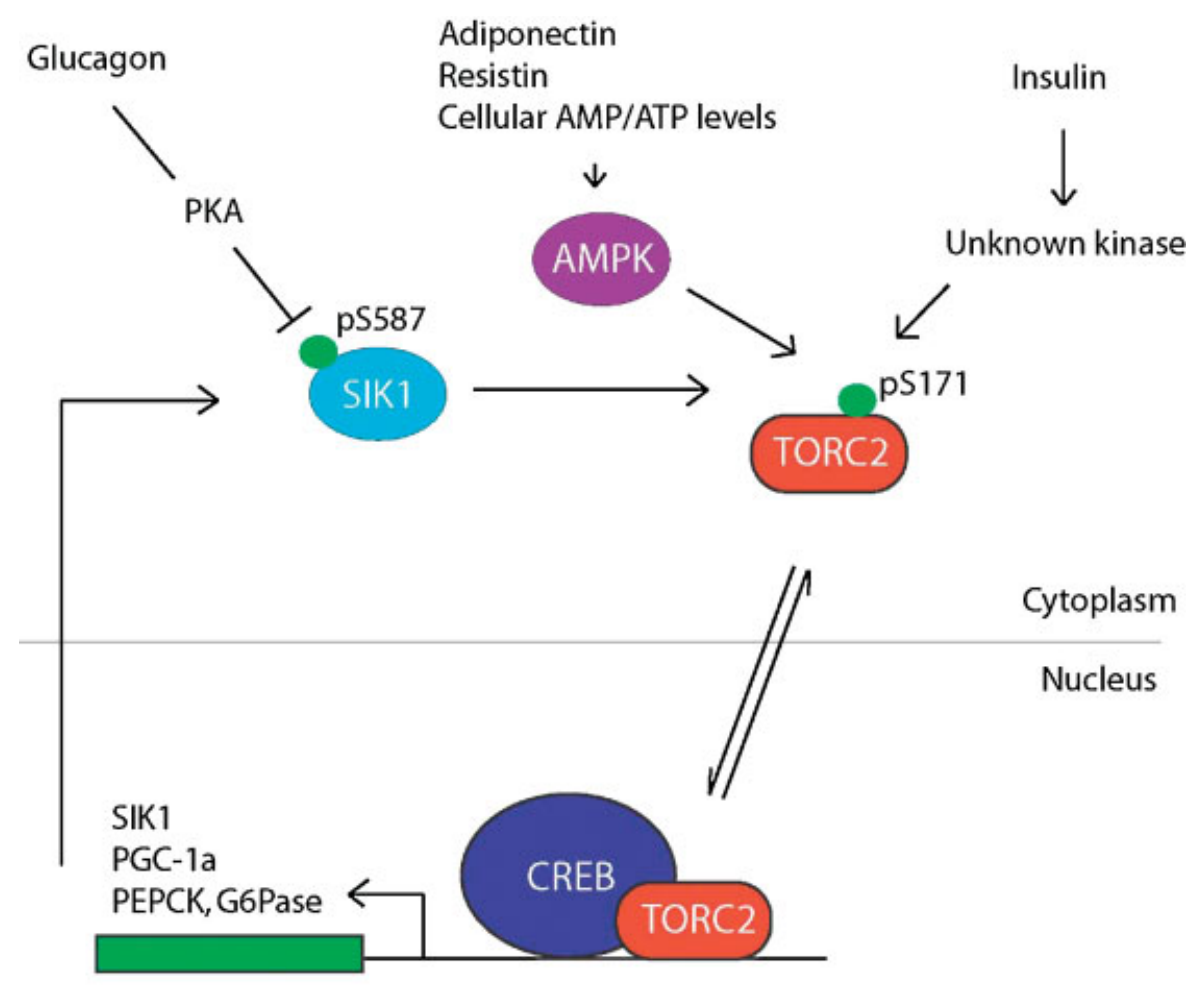

The Gluconeogenic Engine

Figure 1. Cranking up the gluconeogenic engine. During periods of fasting and feeding, the liver responds to secreted hormones by activating signaling pathways that converge on TORC2 to regulate gluconeogenesis. Glucagon promotes the dephosphorylation of TORC2 through the PKA-dependent inhibition of SIKs and, consequently, TORC2 enters the nucleus to activate the gluconeogenic program in concert with CREB. Conversely, insulin- and AMPK-signaling pathways antagonize this process by promoting TORC2 phosphorylation and sequestration in the cytoplasm.

the dephosphorylation of TORC2, likely through the PKA catalyzed phosphorylation and inhibition of SIKs. Conversely, insulin inhibits this process by inducing TORC2 phosphorylation through an unidentified mechanism. The ability of AMPK to phosphorylate TORC2 further adds a level of control that may be modulated by cellular energy status or external stimuli such as the hormones adiponectin and resistin. The identification of TORC2 as a key player in CREB-mediated gluconeogenesis will likely yield new avenues for the design of therapeutics in the treatment of diabetes.

The phosphorylation of TORC2 in response to insulin is reminiscent of the way that the forkhead transcription factors, FOXO1 and FOXA2, are regulated to suppress gluconeogenesis. ${ }^{(17,18)}$ Insulin activates the kinase AKT to phosphorylate these forkhead proteins, leading to their cytoplasmic retention and/or degradation. ${ }^{(19-21)}$ As of yet, the kinase that is responsible for the phosphorylation of TORC2 by insulin remains unknown. Is TORC2 a target of AKT? Are SIKs regulated by insulin? These questions will likely be addressed soon.

\section{References}

1. Koo SH, Flechner L, Qi L, Zhang X, Screaton RA, et al. 2005. The CREB coactivator TORC2 is a key regulator of fasting glucose metabolism. Nature 437:1109-1111.

2. Saltiel AR, Kahn CR. 2001. Insulin signalling and the regulation of glucose and lipid metabolism. Nature 414:799-806.

3. Barthel A, Schmoll D. 2003. Novel concepts in insulin regulation of hepatic gluconeogenesis. Am J Physiol Endocrinol Metab 285:E685-692.

4. Montminy M, Koo SH, Zhang X. 2004. The CREB family: key regulators of hepatic metabolism. Ann Endocrinol (Paris) 65:73-75.

5. Zhou XY, Shibusawa N, Naik K, Porras D, Temple K, et al. 2004. Insulin regulation of hepatic gluconeogenesis through phosphorylation of CREB-binding protein. Nat Med 10:633-637.

6. Herzig S, Long F, Jhala US, Hedrick S, Quinn R, et al. 2001. CREB regulates hepatic gluconeogenesis through the coactivator PGC-1. Nature 413:179-183.

7. Lin J, Handschin C, Spiegelman BM. 2005. Metabolic control through the PGC-1 family of transcription coactivators. Cell Metab 1:361-370.

8. Conkright MD, Canettieri G, Screaton R, Guzman E, Miraglia L, et al. 2003. TORCs: transducers of regulated CREB activity. Mol Cell 12:413-423.

9. lourgenko V, Zhang W, Mickanin C, Daly I, Jiang C, et al. 2003. Identification of a family of CAMP response element-binding protein coactivators by genome-scale functional analysis in mammalian cells. Proc Natl Acad Sci USA 100:12147-12152. 
10. Screaton RA, Conkright MD, Katoh Y, Best JL, Canettieri G, et al. 2004. The CREB coactivator TORC2 functions as a calcium- and CAMPsensitive coincidence detector. Cell 119:61-74.

11. Bittinger MA, McWhinnie E, Meltzer J, lourgenko V, Latario B, et al. 2004. Activation of CAMP response element-mediated gene expression by regulated nuclear transport of TORC proteins. Curr Biol 14:21562161.

12. Sasaki K, Cripe TP, Koch SR, Andreone TL, Petersen DD, et al. 1984. Multihormonal regulation of phosphoenolpyruvate carboxykinase gene transcription. The dominant role of insulin. J Biol Chem 259:1524215251.

13. Wang Z, Takemori H, Halder SK, Nonaka Y, Okamoto M. 1999. Cloning of a novel kinase (SIK) of the SNF1/AMPK family from high salt diet-treated rat adrenal. FEBS Lett 453:135-139.

14. Banerjee RR, Rangwala SM, Shapiro JS, Rich AS, Rhoades B, et al. 2004. Regulation of fasted blood glucose by resistin. Science 303:11951198.

15. Yamauchi T, Kamon J, Minokoshi $Y$, Ito $Y$, Waki H, et al. 2002 Adiponectin stimulates glucose utilization and fatty-acid oxidation by activating AMP-activated protein kinase. Nat Med 8:1288-1295.
16. Shaw RJ, Lamia KA, Vasquez D, Koo SH, Bardeesy N, et al. 2005. The Kinase LKB1 Mediates Glucose Homeostasis in Liver and Therapeutic Effects of Metformin. Science.

17. Puigserver P, Rhee J, Donovan J, Walkey CJ, Yoon JC, et al. 2003. Insulin-regulated hepatic gluconeogenesis through FOXO1-PGC-1alpha interaction. Nature 423:550-555.

18. Wolfrum C, Asilmaz E, Luca E, Friedman JM, Stoffel M. 2004. Foxa2 regulates lipid metabolism and ketogenesis in the liver during fasting and in diabetes. Nature 432:1027-1032.

19. Matsuzaki H, Daitoku H, Hatta M, Tanaka K, Fukamizu A. 2003. Insulininduced phosphorylation of FKHR (Foxo1) targets to proteasomal degradation. Proc Natl Acad Sci USA 100:11285-11290.

20. Wolfrum C, Besser D, Luca E, Stoffel M. 2003. Insulin regulates the activity of forkhead transcription factor Hnf-3beta/Foxa-2 by Aktmediated phosphorylation and nuclear/cytosolic localization. Proc Nat Acad Sci USA 100:11624-11629.

21. Biggs WH 3rd, Meisenhelder J, Hunter T, Cavenee WK, Arden KC. 1999. Protein kinase B/Akt-mediated phosphorylation promotes nuclear exclusion of the winged helix transcription factor FKHR1. Proc Natl Acad Sci USA 96:7421-7426.

\section{BioEssays 28.3}

